\title{
Scale continuum approach in biomechanics: a simple simulation of a microstructural control of tissues' stiffness ${ }^{\text {th }}$
}

\author{
Miroslav Holeček $^{\mathrm{a}, *}$, Olga Červená ${ }^{\mathrm{a}}$, Fanny Poirier ${ }^{\mathrm{b}}$ \\ ${ }^{a}$ Institute of Interdisciplinary Studies, University of West Bohemia in Pilsen, Universitni 22, 30614 Pilsen, Czech Republic \\ ${ }^{\mathrm{b}}$ Laboratoire de Mécanique et d'Acoustique, CNRS, 31 Chemin Joseph Aiguier, 13402 Marseille, France
}

\begin{abstract}
We construct a simple model of an elastic material whose stiffness can be effectively controlled by an appropriately chosen set of microstructural parameters. The model is based on ideas of the scale-dependent continuum description and might play an important role in modelling of muscle tissues in biomechanics.
\end{abstract}

(C) 2002 IMACS. Published by Elsevier Science B.V. All rights reserved.

PACS: 83.10.Dd; 83.20.Bg; 83.80.Lz

Keywords: Scale; Nonlinear elasticity; Microstructure; Muscle tissue

\section{Introduction}

Biomechanics deals with a physical and mechanical modelling of various organs of living organisms to be able to simulate their standard behaviour or various pathological states and problematic situations. A very important problem of this modelling is to find reasonable models of important tissues. The models should be not only realistic but appropriately simple and robust so that the number of "free" parameters (usually very problematic to be determined experimentally) be minimal. A typical tissue whose realistic mechanical model is very needed is the muscle tissue. A striking fact connected with this tissue is its ability to change its mechanical stiffness in an extremely large extent with controlling those changes at a microstructural level. Roughly speaking, the mechanism is as follows: an electrical stimulation triggers some chemical processes whose effect is a change of conformations of some protein molecules. These changes (at the microstructural level) lead to a considerable change of macroscopic mechanical parameters (a muscle becomes very stiff, for example) [1,2].

In this paper, we present a very simple mechanical model in which a big sensitivity of global mechanical parameters on changes of some microstructural parameters occurs. The model is based on the so-called

Expanded version of a talk presented at the MODELLING 2001 (Pilsen, June 2001).

* Corresponding author.

E-mail addresses: holecek@ums.zcu.cz (M. Holeček), poirier@1ma.cnrs-mrs.fr (F. Poirier). 
scale-dependent continuum approach [3-5] in which the continual quantities are supposed to depend on the scale at which they are studied. The approach allows to formulate the continuum theory simultaneously at several scales - an interplay of these scales is that what makes the theory fruitful. In the concrete here, we introduce both a (macroscopic) scale of a standard continuum description and some lower scale at which the microstructural changes are defined. At that ("micro") scale, material "particles"fictitious, artificially chosen parts of the body playing the role of material points of the standard continuum description-become real physical systems which can be described by a thermodynamic description. The strain energy connected with a deformation of the system is thus stored both in elastic bounding among the particles and in inner degrees of freedom of each particle. We model this "inner" energy of particles by using the idea of simple thermodynamic systems which have, in the simplest case, the only one volume variable [6]. To define the change of the volume of individual particles we introduce not only the standard deformation gradient but also that defined at the lower scale. We formulate general features of this model and, as an illustration, we find a simple, two-dimensional example in which a big sensitivity of macroscopic stiffness on microstructural parameters is clearly demonstrated.

\section{Scale continuum description}

The continuum description identifies some parts of a deformable body-usually called the particleswith material points. These points form a continuous structure modelling the body so that an (actual) configuration of the body can be described by a vector function $\boldsymbol{x}: \Omega \rightarrow R^{n}$, i.e. $\boldsymbol{x}(\boldsymbol{X})$. The region $\Omega$ is a bounded subset of $n$-dimensional space $R^{n}$, the so-called reference configuration, so that any point $\boldsymbol{X}$ of $\Omega$ is the label of a material point of the body. The tensor field $\boldsymbol{F}(\boldsymbol{X}), F_{j}^{i}=\partial x^{i} / \partial X^{j}$, is called the gradient of deformation. It describes relative positions of infinitesimally distant particles during the deformation.

In our approach each particle is understood to be a simple thermodynamic system [6]. It means that it can exchange energy with its surrounding and has own state variables (in standard continuum description the energy and state are connected only with a relative position of particles). We ignore thermal effects and thus we can use the simplest thermodynamic description in which the energy of the system is a function of its volume, namely $E(V)$. We will study only elastic deformations and the energy $E$ is understood to be the strain energy stored in the particle as a result of its deformation. Since we work in continuum description we introduce the relative change of volume, $v \equiv V / V_{\mathrm{r}}$, where $V_{\mathrm{r}}$ is the volume of the particle in the reference configuration, and, similarly, we will work with the specific strain energy per unit mass, $e$, instead of $E$. Both quantities are scalar fields, i.e. $v(\boldsymbol{X})$ and $e(\boldsymbol{X})$.

In continuum mechanics, the relative change of local volume is defined as $v_{0}=|\operatorname{det} \boldsymbol{F}|$. However, understanding the particle as an infinitesimal thermodynamic system, $v_{0}$ cannot be a relative change of the volume of this system. We need more such systems (particles) 'surrounding' that to determine $v_{0}$ ( $\boldsymbol{F}$ describes a relative change of positions of particles) [7]. Otherwise speaking, $v_{0}$ is a relative change of the volume of a bigger thermodynamic system including several particles (see Fig. 1). Generally, the field $v$ can be different from $v_{0}$. The crucial step of our approach is based on the idea of the so-called scale-dependent continuum description: we suppose that the deformation gradient is scale-dependent, i.e. $\boldsymbol{F}$ can be understood as a function $\boldsymbol{F}(l)$, where $l$ is a characteristic length-scale. Moreover we assume that there is a scale $l_{m}$ at which the deformation gradient $\boldsymbol{F}\left(l_{m}\right) \equiv \boldsymbol{F}_{m}$ describes inner deformations of individual particles so that the relative change of the particle's volume $v$ is defined as

$$
v=\left|\operatorname{det} \boldsymbol{F}_{m}\right| \text {. }
$$




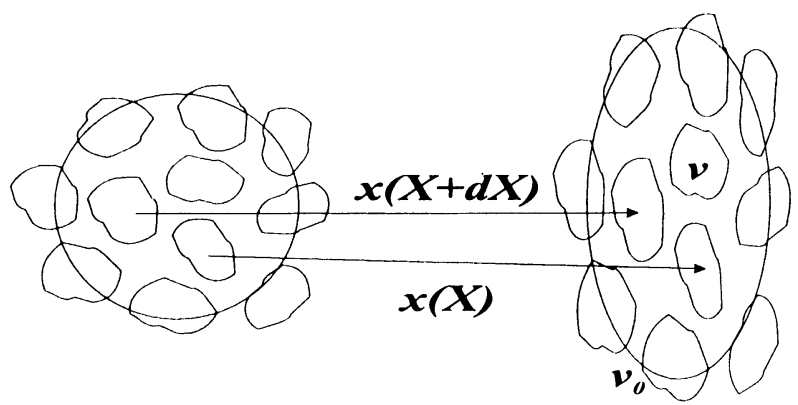

Fig. 1. The relative change of the volume $v_{0}=|\operatorname{det} \nabla \cdot \boldsymbol{x}|$ at the point $\boldsymbol{X}$ is given by displacement of several particles surrounding the point $\boldsymbol{X}$. It implies that it is defined at different scale than the relative change of the volume of individual particles $v$.

In what follows, the symbol $\boldsymbol{F}$ denotes the deformation gradient at some fixed scale $l_{0}, l_{0}>l_{m}$, at which the macroscopic deformation of the body is studied.

The total specific strain energy consists of the deformation energy of individual particles, $e(v)$, and the strain energy coming from interaction of particles. The second one depends not only on the deformation gradient $\boldsymbol{F}$ because there is also an influence of own deformations of the particles. Namely the energy connected with couplings of particles can be increased or decreased only by changing their own deformations (see Fig. 2).

In a linearised approximation this influence may be estimated by a correction $\sim \boldsymbol{M} \cdot\left(\boldsymbol{F}_{m}-\boldsymbol{F}\right)$ to the standard deformation gradient, where the tensor $\boldsymbol{M}$ describes a measure of influence of the shape of individual particles and will be called the microstructural tensor in what follows. Thus, we express the specific (total) strain energy $w$ as a function

$$
w=W\left(\boldsymbol{F}_{e f}, v\right)
$$

where

$$
\boldsymbol{F}_{e f}=\boldsymbol{F}+\boldsymbol{M} \cdot\left(\boldsymbol{F}_{m}-\boldsymbol{F}\right),
$$

and $v$ is given by (1). Let us notice that if there are no scale effects, i.e. $\boldsymbol{F}=\boldsymbol{F}_{m}$, we obtain the strain energy in a form $w=W(\boldsymbol{F}, \operatorname{det} \boldsymbol{F})$, which corresponds with some useful constitutive laws of standard continuum mechanics [8].
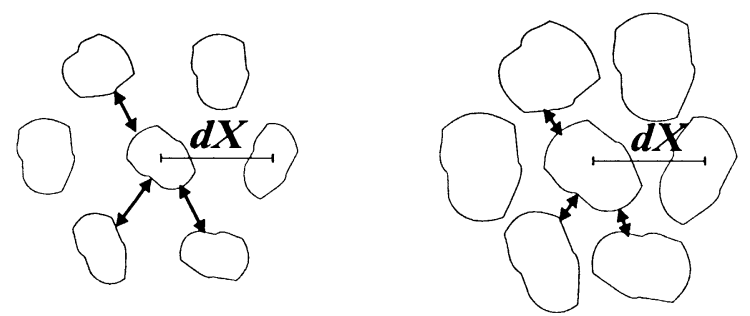

Fig. 2. The interaction energy of particles can be changed by changing the size of particles while the relative position of particles (described by the field $\boldsymbol{F}$ ) is fixed. 
Let us restrict our discussion to elastic materials whose possible configuration minimises the integral

$$
I=\int_{\Omega} w(\boldsymbol{X}) \mathrm{d}^{n} X
$$

within a suitable class of functions $\boldsymbol{x}(\boldsymbol{X})$ respecting boundary conditions. When solving the problem of minimising the integral (4) we can simply suppose that the structure at lower scale $l_{m}$ minimises the local stored energy at any point $\boldsymbol{X}$ while $\boldsymbol{F}$ has a fixed value (we look for the shape of particle minimising the strain energy while positions of surrounding particles are fixed). In other words, finding the minimal value over all possible tensors $\boldsymbol{F}_{m}$ (with fixed $\boldsymbol{F}$ ) we obtain an effective strain energy $w_{0}$ defined at the scale $l_{0}$, i.e.

$$
w_{0}(\boldsymbol{F}, \boldsymbol{X})=\min _{F_{m}} W\left(\boldsymbol{F}_{e f}, \operatorname{det} \boldsymbol{F}_{m}\right) .
$$

\section{Two-dimensional model}

Let us study the deformation of a body which can be defined in two dimensions. Let us suppose that there is a coordinate system in which the tensor $\boldsymbol{F}_{m}$ has a diagonal form, namely

$$
\boldsymbol{F}_{m}=\left(\begin{array}{cc}
\alpha_{1} & 0 \\
0 & \alpha_{2}
\end{array}\right)
$$

( $\alpha_{i}$ may depend on $\boldsymbol{X}$ ). Taking into account that the existence of a minimum of the strain energy with respect to the state of each particle (variable $\boldsymbol{F}_{m}$ ) is supposed (at each point $\boldsymbol{X}$ for any deformation $\boldsymbol{F}$ ) we can approximate the function $w$ around this minimum by a quadratic Taylor approximation. Doing that by supposing that the variable $v=\alpha_{1} \alpha_{2}$ is not "mixed" with the others we obtain

$$
w=g(\boldsymbol{F})+\sum_{i=1,2} a_{i}(\boldsymbol{F})\left(\alpha_{i}-b_{i}(\boldsymbol{F})\right)^{2}+c(\boldsymbol{F})\left(\alpha_{1} \alpha_{2}-d(\boldsymbol{F})\right)^{2} .
$$

Now, solving the minimising problem (5) at the microscopic level (i.e. for each particle individually) we look for an inner particle geometry which minimises the strain energy. It means to solve the conditions

$$
\frac{\partial w}{\partial \alpha_{1}}=0, \quad \frac{\partial w}{\partial \alpha_{2}}=0,
$$

in which the macroscopic deformation variables, stored in the matrix $\boldsymbol{F}$, are understood as fixed parameters. It gives the equations

$$
c \alpha_{1} \alpha_{2}^{2}+a_{1} \alpha_{1}-c d \alpha_{2}-a_{1} b_{1}=0, \quad c \alpha_{1}^{2} \alpha_{2}-c d \alpha_{1}+a_{2} \alpha_{2}-a_{2} b_{2}=0,
$$

and we obtain the solution $\left(\alpha_{1}^{0}, \alpha_{2}^{0}\right)$ being fully dependent on the macroscopic deformation $\boldsymbol{F}$. That is, by putting $\alpha_{i}=\alpha_{i}^{0}(\boldsymbol{F})$ in (7), we obtain the strain energy defined at scale $l_{0}$,

$$
w_{0}=g(\boldsymbol{F})+w_{\text {nonl }}(\boldsymbol{F}) .
$$

The term $w_{\text {nonl }}(\boldsymbol{F}) \geq 0$ plays the role of a nonlinear correction to a standard elasticity (expressed by the term $g$ ). It has a source in own strain energy of particles (expressed by the term $c\left(\alpha_{1} \alpha_{2}-d\right)$ in (7)): if $c=0$ the solution of (9) is

$$
\alpha_{i}=b_{i},
$$




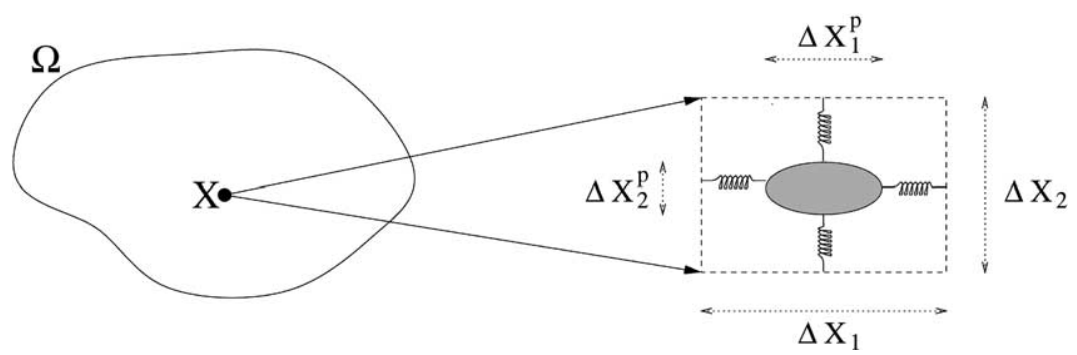

Fig. 3. A simple spring model.

vanishing $w_{\text {nonl }}$ for any macroscopic deformation $\boldsymbol{F}$. If $c \not \equiv 0$ the energy $w_{\text {nonl }}$ is generally nonzero and may play an important role in the macroscopic behaviour of material. The reason is that though (11) cannot be a general solution it can be a very special solution whose occurrence is crucial. Namely, $a_{i}, b_{i}, c, d$ depend not only on $\boldsymbol{F}$ but also on the microstructural tensor $\boldsymbol{M}$ whose elements describe some details of geometric arrangement at scale $l_{m}$. Changes of $\boldsymbol{M}$ thus simulate changes in microstructure. Now, let us image that there exists a microstructural arrangement expressed by the tensor $\boldsymbol{M}=\boldsymbol{M}_{0}$ for which the coefficients $b_{i}, d$ fulfil the following relation:

$$
b_{1}\left(\boldsymbol{M}_{0}\right) b_{2}\left(\boldsymbol{M}_{0}\right)=d\left(\boldsymbol{M}_{0}\right) .
$$

The solution of minimising problem (9) at the "point" $\boldsymbol{M}_{0}$ is then given by the condition (11) which means that the energy $w_{\text {nonl }}$ becomes zero. An (arbitrarily small) change of microstructural tensor (microstructural arrangement) $\boldsymbol{M}_{0}+\delta \boldsymbol{M}$ can lead to a nonzero energy $w_{\text {nonl }}$ which can have a clear macroscopic manifestation. This phenomenon can explain a big sensitivity of material's behaviour on changes in microstructure.

To illustrate it in the concrete, let us construct a two-dimensional spring model consisting of deformable particles interconnected by linear springs as shown in Fig. 3. For simplicity we will suppose that the macroscopic gradient of deformation describing displacements of individual particles has the diagonal form, namely

$$
\boldsymbol{F}=\left(\begin{array}{cc}
\delta_{1} & 0 \\
0 & \delta_{2}
\end{array}\right)
$$

The body deformation has to be considered as a set of deformations,

$$
\Delta X_{1} \rightarrow \delta_{1} \Delta X_{1}, \quad \Delta X_{2} \rightarrow \delta_{2} \Delta X_{2}, \quad \Delta X_{1}^{\mathrm{p}} \rightarrow \alpha_{1} \Delta X_{1}^{\mathrm{p}}, \quad \Delta X_{2}^{\mathrm{p}} \rightarrow \alpha_{2} \Delta X_{2}^{\mathrm{p}},
$$

where $\Delta X_{1}$ and $\Delta X_{2}$ denote the distances of centres of neighbouring particles in the principal deformation directions and $\Delta X_{1}^{\mathrm{p}}$ and $\Delta X_{2}^{\mathrm{p}}$ denote the size lengths of particles in the principal deformation directions. The energy coming from the interaction between neighbouring particles can be approximated as being dependent only on their relative distance $\left(\delta_{i} \Delta X_{i}-\alpha_{i} \Delta X_{i}^{\mathrm{p}}\right) /\left(\Delta X_{i}-\Delta X_{i}^{\mathrm{p}}\right)$. Denoting $\lambda_{i}$ the ratio $\lambda_{i}=$ $\Delta X_{i}^{\mathrm{p}} / \Delta X_{i}$ and taking into account that the strain energy has its minimum at the reference configuration we can choose its quadratic approximation as follows

$$
w=\sum_{i=1,2} \frac{Y}{2}\left(\frac{\delta_{i}-\alpha_{i} \lambda_{i}}{1-\lambda_{i}}-1\right)^{2}+K\left(\alpha_{1} \alpha_{2}-1\right)^{2}+g\left(\delta_{1}, \delta_{2}\right),
$$


where $Y$ and $K$ are physical parameters. Let us notice that the microstructural tensor is given as $M_{i j}=$ $-\lambda_{i}\left(1-\lambda_{i}\right)^{-1} \delta_{i j}$. The relation (12) is fulfilled when

$$
\lambda_{1}\left(\delta_{2}-1\right)+\lambda_{2}\left(\delta_{1}-1\right)=\delta_{1}+\delta_{2}-\delta_{1} \delta_{2}-1 .
$$

Thus, for any couple of parameters $\lambda_{i}^{(0)}$ fulfilling this relation we obtain a zero energy $w_{\text {nonl }}$. It is worth stressing that this relationship depends only on $\delta_{i}$ and for any macroscopic deformation $\delta_{i}$ we can find such a couple of microstructural parameters. Numerical solution of the microscopic minimising problem (9) enables us to plot the dependence of $w_{\text {nonl }}$ on microstructural parameters as shown in Fig. 4.

We see that just a small change in the microstructural parameters can lead to an important increase or decrease of the strain energy. This change then considerably influences the macroscopic deformation of the system. Taking into account the term $g\left(\delta_{1}, \delta_{2}\right)$ in $(15)$ we have the system having some standard elasticity (not dependent on the microstructure) and an additional nonlinear term strongly dependent on the

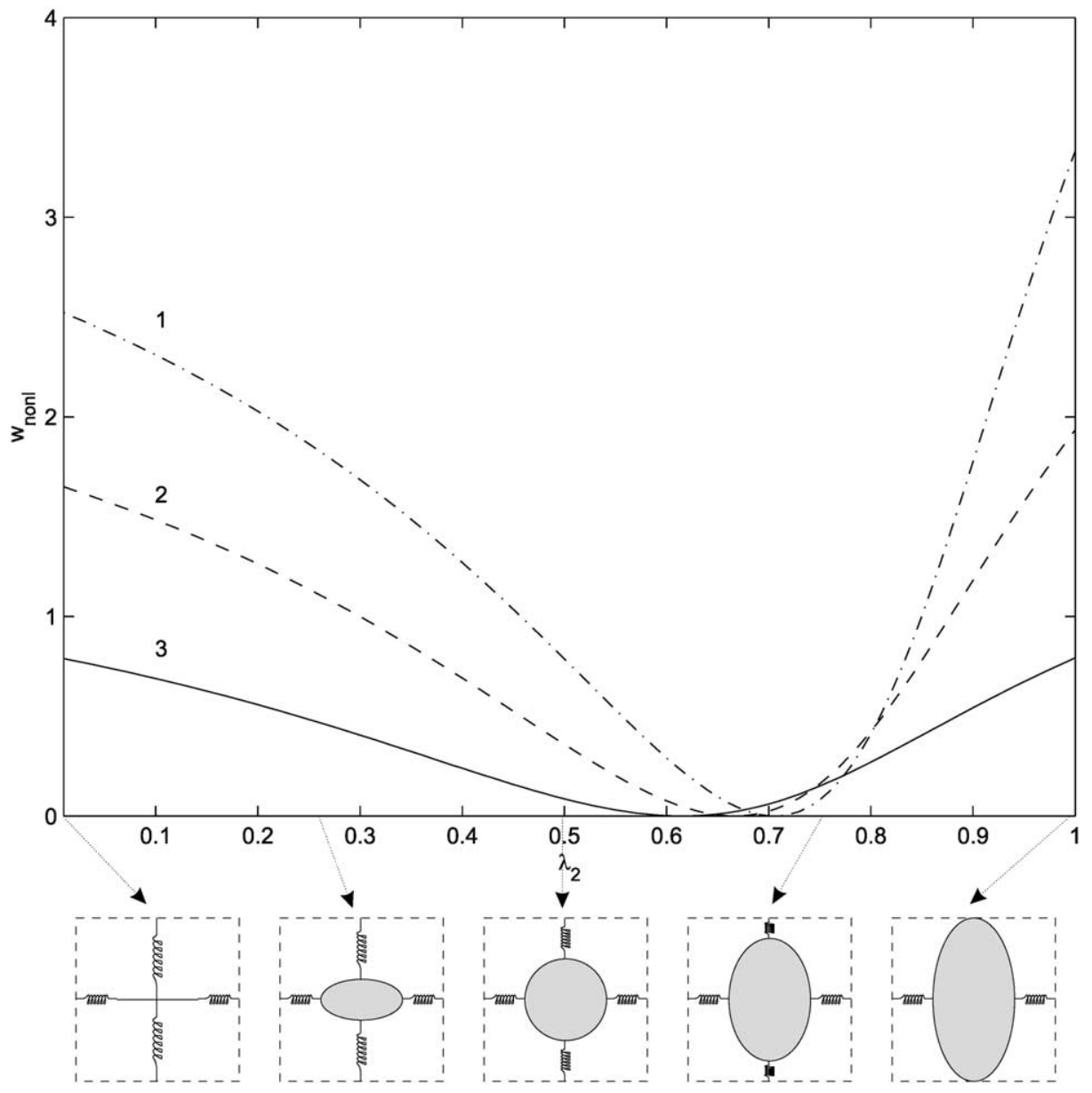

Fig. 4. Relationship between the correction part $w_{\text {nonl }}$ of the specific strain energy and the microstructural parameter $\lambda_{2}$ for fixed values of $\lambda_{1}\left(\lambda_{1}=0.5\right), Y(Y=0.3 \mathrm{MPa}), K(K=0.36 \mathrm{MPa})$ in the case of macroscopically incompressible materials $\left(\delta_{1}=\delta_{2}^{-1}\right)$ for three cases of macroscopic deformations: $\delta_{1}=1.7($ plot 1$), \delta_{1}=1.5($ plot 2$), \delta_{1}=1.3($ plot 3$)$. 
microstructure. If $g\left(\delta_{1}, \delta_{2}\right)$ is small comparing to the second term (if states are far from the "point" (16)) we can expect big changes in deformations of the system when some microstructural changes happen. In the concrete, let us imagine that the microstructural parameters are "tuned" so that the material is near the point (16). Then only the standard elasticity described by the term $g$ plays a role and the material is in configuration minimising this energy with respect to boundary conditions (e.g. a prescribed pressure). Let us suppose that this energy is low enough so that the pressure leads to a big deformation of the material. Now, let some inner changes in the material lead to a change in microstructure sending the microstructural parameters far from the point (16). If the deformation did not change it would lead to an important increase of the elastic energy (due to the term $w_{\text {nonl }}$ ), which is impossible because no macroscopic supply of energy happened (the external pressure is the same). Thus, the material has to considerably decrease its deformation-it behaves as if its macroscopic stiffness sharply increased. This phenomenon resembles qualitative processes in muscle tissues: an extremal change in macroscopic mechanical parameters due to changes of conformations of protein chains within muscle cells — the passive state comes to the stimulated one [1].

\section{Conclusion}

We use the scale continuum approach in description of an elastic body whose actual state is characterised by a minimal value of its elastic energy. Understanding each "particle" (a material point of the standard continuum description) as a simple thermodynamic system we can suppose that the minimal value of the global energy means minimisation of own energy of all particles too. The state of each particle is interconnected with a (macroscopic) deformation by a set of microstructural parameters describing a detailed geometric arrangement of individual particles and thus we obtain the model of an elastic material whose behaviour can be controlled by changing these microstructural parameters.

To illustrate the method we have constructed the two-dimensional model modelling the strain energy of particles by a quadratic function. An important feature of this model is the emergence of an additional term $w_{\text {nonl }}$ which gives a nonlinear correction to a standard elasticity. It essentially depends on some microstructural parameters (e.g. $\lambda_{i}$ in the model (15)) and is very sensitive on the change of these parameters. If there is a possibility of varying these parameters near the "point" where the term $w_{\text {nonl }}$ vanishes, the material can exhibit a broad spectrum of material properties (e.g. its stiffness can vary considerably). A concrete example of such a "controlling" point is shown on a simple spring model (though using linear springs the model gives nonlinear behaviour).

The model enables us to simulate controlling of a macroscopic model by changes of microstructural parameters. It is a promising step because such a behaviour exhibits some "materials" in living nature. The muscle tissue, for example, varies largely its stiffness by doing some changes at the microstructural levellarge protein molecules like actin and myosin change their conformations (very roughly resembling the change of proportions of springs in Fig. 3). The question whether this way of using the scale continuum description can lead to a more realistic models of the muscle tissue is a challenging problem for further progress.

\section{Acknowledgements}

The work has been supported by the Czech Ministry of Education, Project No. MSM230000009. 


\section{References}

[1] Y.C. Fung, Biomechanics: Mechanical Properties of Living Tissues, Springer, Berlin, 1993.

[2] J. Keener, J. Sneyd, Mathematical Physiology, Interdisciplinary Applied Mathematics, Springer, Berlin, 1998.

[3] M. Holeček, Heat conduction equation as a limit of scale-dependent hydrodynamics, Physica A 183 (1992) $236-246$.

[4] M. Holeček, Scale-dependent space averaging, University of West Bohemia, Pilsen, 1996 (Preprint No. 91).

[5] M. Holeček, P. Krákora, Scale-dependent continuum models in biomechanics, J. Mech. Eng. 52 (2001) $175-187$.

[6] E.H. Lieb, J. Yngvason, The physics and mathematics of the second law of thermodynamics, Phys. Reports 310 (1999) 1-96.

[7] M. Holeček, Model of critical behaviour in elasticity, J. Computat. Appl. Math. 63 (1995) 271-275.

[8] J.E. Marsden, T.J.R. Hughes, Mathematical Foundations of Elasticity, Prentice-Hall, Englewood Cliffs, New Jersey, 1983. 